\title{
LEVANTAMIENTO PLANIMÉTRICO Y ANÁLISIS LIQUENOMÉTRICO EN EL SITIO CAMPO DE CHENQUES, COSTA NORTE DE SANTA CRUZ (PATAGONIA ARGENTINA)
}

\author{
PLANIMETRIC SURVEY AND LICHENOMETRIC ANALYSIS IN CAMPO DE \\ CHENQUES SITE, NORTHERN COAST OF SANTA CRUZ \\ (ARGENTINIAN PATAGONIA)
}

\author{
Leandro Zilio ${ }^{1,2}$, Heidi Hammond ${ }^{1,2}$ y Alicia S. Castro ${ }^{2}$
}

\begin{abstract}
Se presentan los resultados de dos vías metodológicas desarrolladas en el sitio Campo de Chenques, las que resultan novedosas para la arqueología de cazadores recolectores que habitaron la Patagonia. El sitio se ubica en la costa atlántica de la provincia de Santa Cruz, Argentina, y presenta una concentración de estructuras de entierro humanas de tipo chenque.

Se realizó el levantamiento planimétrico de las estructuras de rocas que conforman el sitio a partir del uso de un GPS diferencial. Además, se desarrollaron estudios liquenométricos con el fin de obtener cronologías de las estructuras. Para ello se midieron líquenes del género Rhizocarpon subgénero Rhizocarpon que crecen sobre las rocas.

A partir de estos estudios se realizó un plano georreferenciado del sitio, se determinó el número y morfología de las estructuras y se obtuvo por liquenometría una edad mínima para la construcción de las mismas de 770 años, aproximadamente, la que es concordante con las edades radiocarbónicas obtenidas para el sitio. De este modo, Campo de Chenques es el contexto arqueológico más antiguo de Patagonia en el que se han aplicado análisis liquenométricos hasta el momento.

Los resultados se discuten en relación con la dinámica de ocupación del área por las poblaciones cazadoras recolectoras durante el Holoceno tardío.
\end{abstract}

Palabras claves: levantamiento planimétrico, liquenometría, GPS diferencial, Chenques, Patagonia.

This paper presents the results obtained from the planimetric survey and lichenometric analyses conducted for the first time in the hunter-gatherer archaeology of Patagonia. Campo de Chenques site is a concentration of chenque-type human burial structures located on the Atlantic coast of the province of Santa Cruz, Argentina. A planimetric survey of the structures that make up the site was performed using a differential GPS. In addition, lichenometric studies were undertaken in order to obtain minimum estimated ages for the structures. To this end, lichen genus Rhizocarpon (subgenus Rhizocarpon) that grow on the rocks were measured. Based on these studies, a georeferenced map of the site was made, the number and morphology of the structures were determined, and a minimum age of approximately 770 years for the chenque construction was obtained by lichenometry, which is consistent with radiocarbon ages obtained for the site. Thus, Campo de Chenques becomes the oldest archaeological context of Patagonia in which a lichenometric technique was applied for the first time.

The results are discussed in relation to the dynamics of occupation of the area by hunter-gatherer populations during the late Holocene.

Key words: Planimetric survey, lichenometry, differential GPS, Chenques, Patagonia.

Al sur de la localidad arqueológica Punta Medanosa, en la costa norte de la provincia de Santa Cruz (Patagonia argentina), se encuentra el sitio Campo de Chenques (Figura 1). Este presenta una concentración de estructuras de entierro de tipo chenque emplazadas cercanas a la línea de costa actual (Castro y Moreno 2000). Hasta el momento se dispone de dos dataciones radiocarbónicas que ubican al sitio en el Holoceno Tardío final (Zilio 2015; Zilio et al. 2013).

Los chenques son la forma de inhumación humana más representada en toda la Patagonia durante el Holoceno Tardío. Se han identificado contextos tanto individuales como múltiples, los cuales presentan como característica distintiva una cubierta de rocas sueltas, a modo de montículo,

1 CONICET. Consejo Nacional de Investigaciones Científicas y Técnicas. Av. Rivadavia 1917 (C1033AAJ), Buenos Aires, Argentina.

2 División Arqueología, Facultad de Ciencias Naturales y Museo, Universidad Nacional de La Plata. Museo de La Plata, Paseo del Bosque s/nº (1900), La Plata, Argentina. leandrozilio@yahoo.com.ar; heidihammondunlp@gmail.com; acastro52@gmail.com 


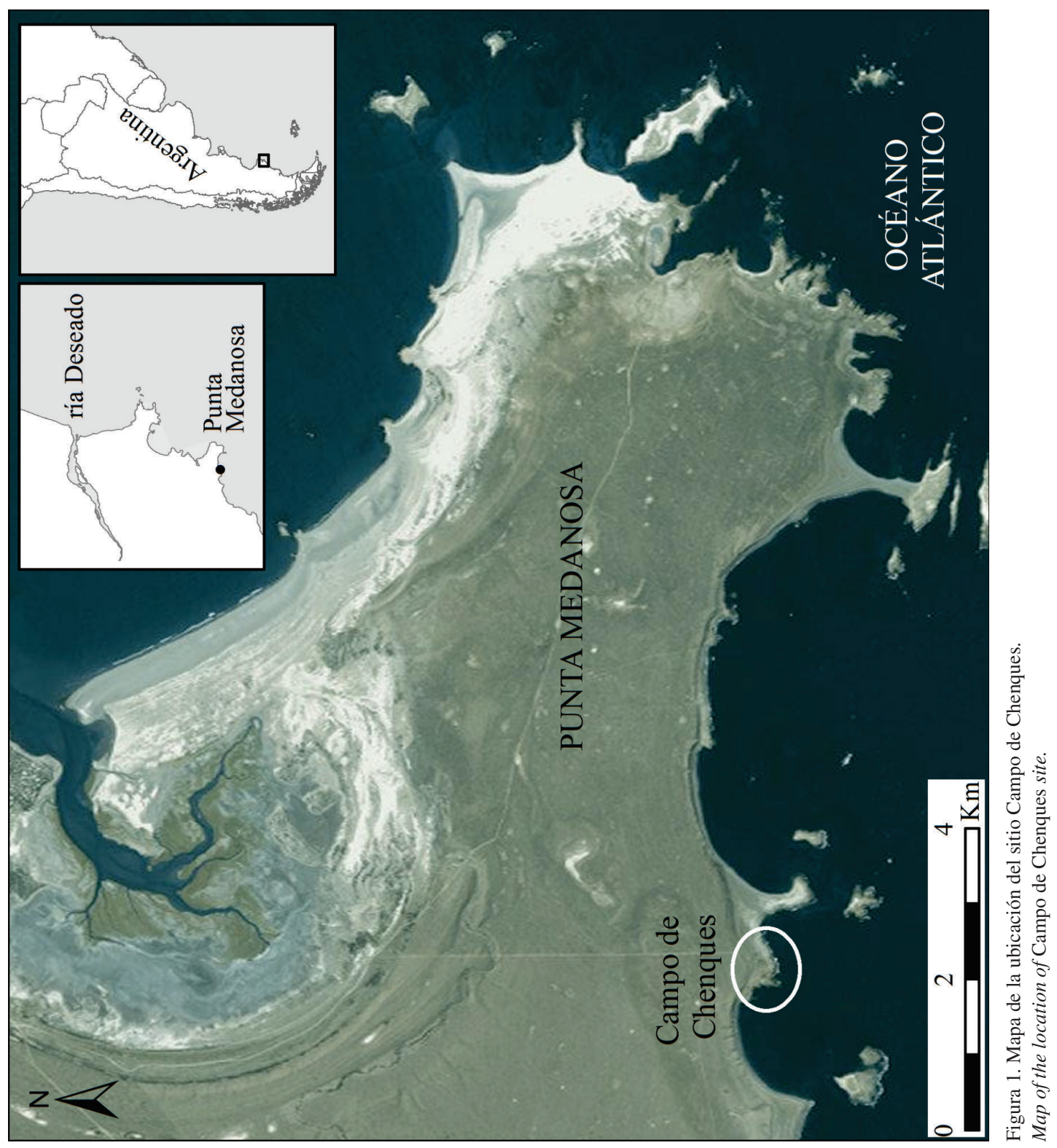


depositada por sobre el/los individuos inhumados (Zilio 2013).

Campo de Chenques constituye un sitio arqueológico de características singulares en Patagonia, debido al gran número de estructuras de entierro de cazadores recolectores agrupadas en una superficie acotada del paisaje (Castro y Moreno 2000; Zilio 2015).

Los objetivos de este trabajo son reconocer la antigüedad de las estructuras de piedra en el sitio Campo de Chenques, evaluar la morfología de las mismas y analizar su distribución espacial intrasitio. Debido a que las estructuras presentan signos de saqueo, la tarea de obtener cronologías absolutas constituye una labor dificultosa; por ello se decidió implementar la técnica de liquenometría a partir del análisis de líquenes del género Rhizocarpon subgénero Rhizocarpon, los cuales fueron identificados en el sitio.

La liquenometría es una técnica de datación que consiste en el uso de líquenes que crecen sobre una superficie para estimar su edad de exposición (Garibotti et al. 2011). El desarrollo de líquenes del género Rhizocarpon subgénero Rhizocarpon es radial, por lo que conociendo su tasa anual de crecimiento es posible estimar la edad de un ejemplar a partir de la medición de su diámetro.

El sitio Campo de Chenques presenta dificultades a la hora de mapear cada una de las estructuras de piedra debido a la contigüidad espacial de las mismas. Por ello, se decidió realizar un levantamiento planimétrico del sitio a partir del empleo de un GPS diferencial, con el objeto de generar un plano preciso. Se registró la localización de las estructuras, la línea de alta marea y curvas de nivel. El plano elaborado permitió conocer el número de estructuras y analizar su variabilidad morfológica.

El GPS diferencial es una herramienta que recibe y procesa información de satélites, además de datos adicionales procedentes de una estación terrestre situada en un lugar cercano y reconocido. Esta información complementaria permite corregir las inexactitudes que se puedan introducir en las señales que el receptor recibe de los satélites (Amado Reino 1999).

Se pretende que estos estudios aporten nuevos conocimientos para discutir la dinámica de ocupación humana durante el Holoceno Tardío en la costa norte de la provincia de Santa Cruz.

\section{Antecedentes del Sitio Campo de Chenques}

A comienzos de la década de 1950 Menghin y Bórmida (s/f) indican la presencia de gran cantidad de enterratorios en la localidad arqueológica Punta Medanosa (Figura 1). En la costa sureste de esta localidad los autores describen un sitio al cual denominan Campo de Chenques. Allí señalan la existencia de unos 30 túmulos funerarios situados en una franja de $300 \mathrm{~m}$ de largo por 60 a $80 \mathrm{~m}$ de ancho, destacan que los enterratorios se encuentran frente a una playa compuesta por lajas porfídicas y que las mismas fueron utilizadas para la construcción de los túmulos. Caracterizan a estos chenques como amontonamientos de lajas, algunas de gran tamaño, dispuestas a nivel del piso y superpuestas al cadáver que fue depositado directamente sobre la superficie del suelo.

Menghin y Bórmida (s/f) plantean que la mayoría de las estructuras se encuentran intactas y que pudieron extraer cuatro esqueletos incompletos de adultos y el de un niño. Destacan que a este último se lo halló en una estructura más pequeña. La posición de dos de los individuos era decúbito lateral extendido (derecho e izquierdo) dirigiendo la cabeza uno hacia el mar (sudeste) y el otro hacia el noroeste. También expresan que ningún objeto de ajuar fue hallado en el sitio.

Bórmida en su trabajo "Los Antiguos Patagones", describe dos de los cráneos excavados en Campo de Chenques. El autor plantea que los cráneos pertenecen a un individuo femenino y otro masculino, ambos adultos, con deformación craneana planofrontal y en posición decúbito dorsal extendida (Bórmida 1953-1954:30).

En el marco de las tareas de investigación llevadas a cabo por Alicia Castro, en 1999 se realizó un relevamiento cualitativo y un registro detallado de las estructuras de entierro del sitio, aunque sin precisión en cuanto a la localización de los chenques en el paisaje (Castro y Moreno 2000; Castro et al. 2001). Se determinó la presencia de 86 estructuras (Castro y Moreno 2000; Castro et al. 2001). Como se observa, este número es mayor al mencionado con anterioridad por Menghin y Bórmida (s/f).

Castro y Moreno (2000) sugirieron que esta cantidad de enterratorios podría deberse a causas como la ocupación reiterada de este sector de costa; que el sitio haya sido ocupado por grupos 
grandes de personas y durante períodos extensos de tiempo, dando lugar a que sucedan situaciones de muerte; o la posibilidad de que sean enterratorios secundarios, lo que en ese caso además implicaría la existencia de razones particulares para la elección de un mismo lugar de inhumación (Castro y Moreno 2000:230).

Durante tareas de campo realizadas por Castro en 2000 se recuperaron escasos restos óseos humanos expuestos en superficie en cinco estructuras afectadas por saqueo, las cuales posteriormente se denominaron $\mathrm{CCH}-2, \mathrm{CCH}-3, \mathrm{CCH}-4, \mathrm{CCH}-5$ y CCH-6 (Zilio 2015).

Con el objetivo de corroborar que los montículos de piedras corresponden a entierros humanos, en el 2001 se llevaron a cabo sondeos en seis estructuras (Castro 2001). Se buscó registrar la presencia de restos óseos humanos con el fin de confirmar su carácter de enterratorio, aunque los mismos no fueron recolectados. En tres estructuras se hallaron restos humanos que permitieron contrastar positivamente su calidad de entierro. En otra se identificó una raedera de sílex sin asociación contextual y en las dos restantes no se recuperó material. Las excavaciones fueron de pequeñas dimensiones, por ello la información es parcial, pero suficiente para considerar a las estructuras como entierros humanos en contra de la idea de montículos de piedras con otros fines no funerarios.

En 2004, Goñi y colaboradores excavaron una estructura denominada CCH-1 (Goñi et al. 2005). Este chenque presentaba evidencias de haber sido abierto, ya que se observaban tanto rocas removidas de su posición original como huesos humanos aflorando en superficie. Las evidencias recuperadas permitieron establecer que se trataba de un entierro primario individual, cuya integridad anatómica estaba alterada por saqueo, particularmente la porción superior del tronco, extremidades superiores, cintura escapular y cráneo. El esqueleto ocupaba la parte central de la estructura, en una pequeña depresión, posiblemente resultado de una excavación intencional. La posición del cuerpo era decúbito dorsal extendida. Los resultados bioantropológicos indicaron que corresponde a un individuo masculino aproximadamente entre 30 y 40 años de edad. Solo se registraron modificaciones patológicas en los cuerpos de las vértebras lumbares (labiaciones). A partir de muestras óseas de este individuo se llevaron a cabo determinaciones de los valores de $\delta^{13} \mathrm{C}$ (colágeno) y de la relación $87 \mathrm{Sr} / 86 \mathrm{Sr}$. El valor de $\delta^{13} \mathrm{C}$ obtenido $(-16,3 \%$ ) indicaría una dieta probablemente mixta, basada en la incorporación de recursos terrestres y marinos (Moreno et al. 2011). Mientras que el análisis de la relación $87 \mathrm{Sr} / 86 \mathrm{Sr}$ arrojó un resultado de 0,708954 , el que es difícil de interpretar debido a la ausencia de otros valores locales de referencia. Sin embargo, se encuentra próximo al valor medio del agua oceánica $(0,7092)$, lo que podría sugerir que muy probablemente el individuo transcurrió los últimos años de su vida en ambientes costeros (Goñi et al. 2005).

Los relevamientos realizados por Zilio en el 2013 permitieron identificar escasos restos óseos humanos en superficie en tres estructuras alteradas por saqueo (CCH-13P, CCH-31P y CCH-40P; Zilio 2015).

Recientemente se llevaron a cabo dataciones radiocarbónicas en restos óseos humanos recuperados en el sitio, así como análisis de isótopos estables de $\delta^{13} \mathrm{C}$ y $\delta^{15} \mathrm{~N}$ (Zilio 2015; Zilio et al. 2013; Tabla 1). Los restos humanos del entierro CCH-1, mencionado anteriormente, fueron datados en $890 \pm 60$ a.p. (LP-2778, colágeno de hueso, $\delta^{13} \mathrm{C}=-16,30 \%$ ). Además, sobre restos óseos de la estructura $\mathrm{CCH}-2$ se realizó otra datación radiocarbónica y se obtuvo una edad de $1.120 \pm 50$ a.p. (LP-3046; colágeno de hueso, $\delta^{13} \mathrm{C}=-16,05 \%$ ).

En la Tabla 1 se resumen los valores de isótopos estables obtenidos, los que en todos los casos indican un componte marino en la dieta. En la totalidad de las muestras estudiadas la relación $\mathrm{C} / \mathrm{N}$ medida sobre el colágeno es de entre 3,0 y 3,4 (Tabla 1). Estos valores se ubican dentro del rango aceptado para muestras sin alteración diagenética (De Niro 1985). Los resultados de isótopos estables para el sitio Campo de Chenques presentan una media de $-14,21 \pm 2,36 \%$ para $\delta^{13} \mathrm{C}$ (mediana $-14,26$ ) y $17,91 \pm 3,36 \%$ para $\delta^{15} \mathrm{~N}$ (mediana 17,65 ).

Se resalta que el promedio de valores de $\delta^{15} \mathrm{~N}$ obtenidos de las muestras de individuos de Campo de Chenques se encuentra entre los más altos observados en Patagonia (Barberena 2002; Borrero et al. 2001; Favier Dubois et al. 2009; Gómez Otero 2006; Guichón et al. 2001; Suby et al. 2009; Tessone 2010; Yesner et al. 1991; Zilio et al. 2014). Incluso la muestra con los valores más altos de $\delta^{13} \mathrm{C}$ y $\delta^{15} \mathrm{~N}$ de toda la Patagonia proviene de este sitio $(\mathrm{CCH}-$ 40P; Tabla 1). Otros contextos donde también se han registrado valores elevados de $\delta^{15} \mathrm{~N}$ se ubican 
Tabla 1. Síntesis de los análisis de muestras óseas humanas recuperadas en el sitio Campo de Chenques. Synthesis of analyses on human bone samples recovered at Campo de Chenques site.

\begin{tabular}{|c|c|c|c|c|c|}
\hline Estructura & CCH-1 & $\mathrm{CCH}-2$ & CCH-13P & CCH-31P & $\mathrm{CCH}-40 \mathrm{P}$ \\
\hline Sexo & Masculino & $\mathrm{s} / \mathrm{d}$ & $\mathrm{s} / \mathrm{d}$ & s/d & $\mathrm{s} / \mathrm{d}$ \\
\hline Edad & Adulto medio & Adulto & Adulto & Adulto & Adulto \\
\hline Edad ${ }^{14} \mathrm{C}$ & $890 \pm 60$ & $1.120 \pm 50$ & - & - & - \\
\hline $\begin{array}{l}\text { Edad cal. años a.p. } \\
\qquad(2 \sigma)^{1}\end{array}$ & $906-672$ & $1.089-820$ & - & - & - \\
\hline Mediana (cal a.p.) & 761 & 983 & - & - & - \\
\hline Código & LP 2778 & LP 3046 & - & - & - \\
\hline$\delta^{13} \mathrm{C}_{\text {col. }}$ & $-16,30$ & $-16,05$ & $-14,08$ & $-14,26$ & $-10,38$ \\
\hline$\delta^{15} \mathrm{~N}$ & 14,98 & 15,42 & 17,65 & 18,08 & 23,43 \\
\hline $\mathrm{C} / \mathrm{N}$ & 3,2 & 3,2 & 3,3 & 3,3 & 3,3 \\
\hline Muestra & Metapodiano & Frag. de húmero & Frag. de calcáneo & Frag. de rótula & Frag. diaf. radio \\
\hline Código & EILAB 195948 & AIE 35314 & AIE 35411 & AIE 35406 & AIE 35390 \\
\hline Referencia & $\begin{array}{l}\text { Goñi et al. 2005; } \\
\text { Moreno et al. 2011; } \\
\text { Zilio et al. } 2013\end{array}$ & Zilio 2015 & Zilio 2015 & Zilio 2015 & Zilio 2015 \\
\hline
\end{tabular}

en la costa norte de Chile (Santana Sagredo et al. 2015), la costa sur de Perú (Tomczak 2003) y la costa de Brasil (Colonese et al. 2014), así como en las poblaciones históricas de esquimales de Alaska, cuya dieta estaba compuesta principalmente por mamíferos marinos, y los indios Haida y Tlingit de la costa noroeste de Estados Unidos, los cuales dependían de manera fundamental de la pesca de salmones (Schoeninger et al. 1983).

\section{Metodología}

\section{Levantamiento planimétrico}

El relevamiento planimétrico se realizó a partir del empleo de un GPS diferencial, marca GTR-A BT (Figura 2). El mismo consta de dos receptores, uno de ellos estático, que se situó en un punto con coordenadas de ubicación conocida (estación de referencia). Este receptor almacena la información proporcionada por los satélites durante el transcurso de las mediciones de campo y calcula el error con el que cuentan las señales de cada uno de los satélites visibles en cada instante de medición. El cálculo se realiza contrastando los valores obtenidos con la posición real de la estación de referencia. El segundo de los receptores es la unidad portátil que se empleó en el sitio, con la que a su vez se genera un archivo de valores no corregidos. La combinación de la información de los dos receptores da como resultado un conjunto de medidas corregidas (Amado Reino 1999).

Las tareas desarrolladas en el campo consistieron inicialmente en la localización de la estación de referencia en un lugar conocido, ubicado dentro del rango de cobertura del instrumental, con el fin de que la señal sea captada por la unidad portátil. Posteriormente se recorrió a pie con dicha unidad todo el terreno que ocupa el sitio, se registró la línea de alta marea y el contorno de cada una de las estructuras (Figura 2). A partir del empleo del instrumental se generó un archivo compuesto por una nube de puntos, los cuales luego se tradujeron en curvas de nivel. Paralelamente, durante las tareas de campo se relevaron las medidas de las estructuras con una cinta métrica.

Para un análisis espacial de las estructuras se generó un mapa de localización y de densidad mediante el empleo de Sistemas de Información Geográfica (SIG) utilizando el software ArcMap 10.1. Se dibujó el contorno de cada una de las estructuras y se corroboraron los diámetros a partir de las medidas obtenidas con la cinta métrica. Se trabajó con el estimador de Densidad Kernel, a partir del cual se calcula la densidad de las entidades registradas según la presencia o no de entidades en la vecindad, utilizando el método del "Vecino más cercano" (Moreno Jiménez 2005). 


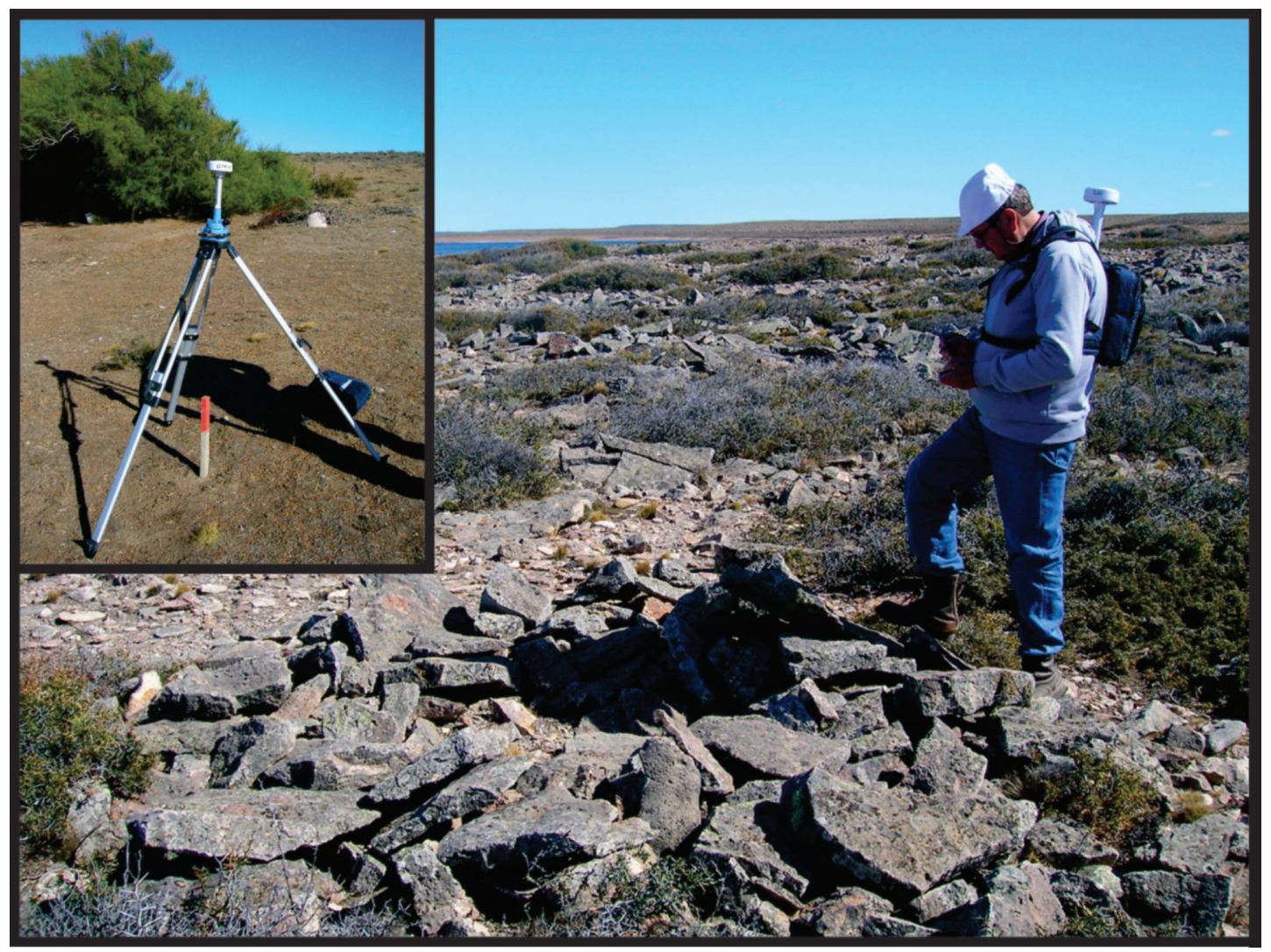

Figura 2. Relevamiento de las estructuras de rocas en el sitio Campo de Chenques mediante GPS diferencial. Recuadro: Estación de referencia. Fotografía general: Unidad portátil.

Survey of the rocks structures at Campo de Chenques site by differential GPS. Box: Reference station. General photography: Portable unit.

\section{Liquenometría}

La liquenometría es una técnica de datación relativa que consiste en el uso de líquenes que crecen sobre una superficie para estimar la edad de exposición (Borrazzo y Garibotti 2014; Borrero et al. 2011; Garibotti et al. 2011; Morano-Büchner y Aravena 2013). Los líquenes son colonizadores tempranos de superficies rocosas; su crecimiento es lento, radial y son organismos longevos, por lo cual se consideran organismos especialmente útiles para datar superficies de edad desconocida (Borrero et al. 2011). Una de las ventajas de esta técnica es su bajo costo, ya que las mediciones de los líquenes en las rocas se realizan utilizando una cinta flexible, calibre o mediante series fotográficas (Borrero et al. 2011). Además esta es una técnica no intrusiva.
Diferentes muestras de líquenes fueron colectadas en el sitio y enviadas para su análisis al LEMIT (Laboratorio de Entrenamiento Multidisciplinario para la Investigación Tecnológica), con el objetivo de corroborar su filiación taxonómica al género Rhizocarpon subgénero Rhizocarpon. La Dra. Rosato confirmó que efectivamente los ejemplares correspondían al liquen mencionado.

Los líquenes del subgénero Rhizocarpon presentan un color amarillo verdoso y poseen un anillo negro (protalo), característica que facilita su reconocimiento, permite diferenciar individuos aislados de talos coalescentes y verificar si un individuo está completo (Borrero et al. 2011; Garibotti et al. 2011; Figura 3).

Con el fin de establecer una edad mínima de construcción de los entierros en Campo de Chenques, se midieron los líquenes del subgénero 


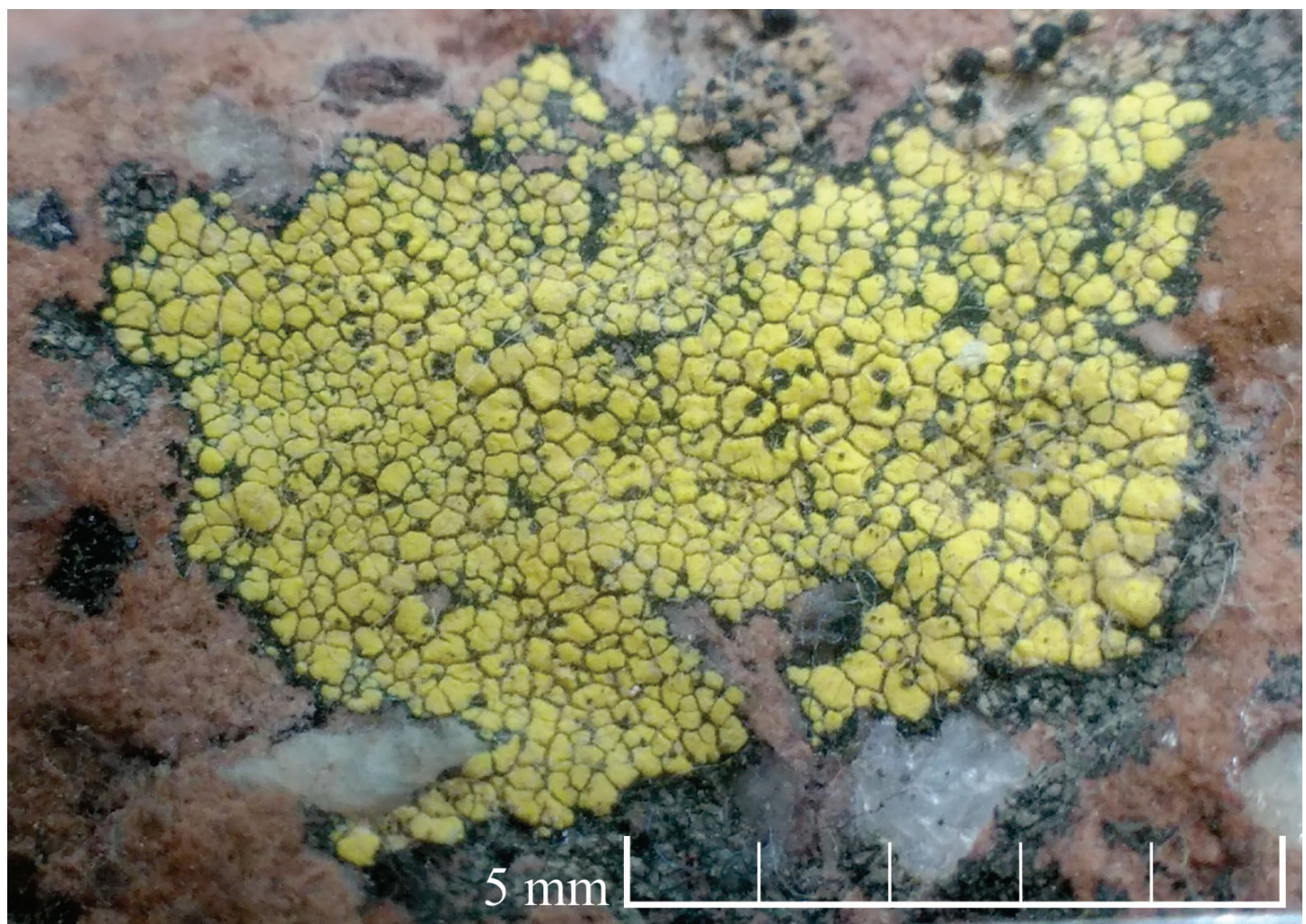

Figura 3. Espécimen de liquen del género Rhizocarpon subgénero Rhizocarpon. Specimen of lichen genus Rhizocarpon subgenus Rhizocarpon.

Rhizocarpon identificados en las estructuras (Figura 3). A partir de la estimación de la tasa de crecimiento de líquenes de distintos tamaños es posible trazar la curva de crecimiento que representa la relación entre la edad y el diámetro de los líquenes para un rango amplio de tamaños y edades (Garibotti et al. 2011:88).

Se emplearon distintas aproximaciones para datar las estructuras utilizando los especímenes de Rhizocarpon Rhizocarpon. Estas aproximaciones fueron desarrolladas por Borrero et al. (2011) y Garibotti et al. (2011). Por un lado, se llevó a cabo la aproximación tradicional, la cual consiste en medir el diámetro de los líquenes de mayores dimensiones que crecen en la superficie de las rocas. Conociendo la tasa de crecimiento radial del liquen es posible estimar la edad mínima de exposición de la superficie a partir del diámetro del espécimen más grande encontrado en el sitio. Según Borrero et al. (2011:200), este método en arqueología es cuestionable debido a la dificultad de determinar si el liquen más grande encontrado se estableció posteriormente a la construcción de la estructura arqueológica, o es un individuo que se estableció previamente en la roca y sobrevivió a su traslado. Este problema puede ser abordado utilizando una aproximación liquenométrica poblacional. El análisis de las distribuciones de frecuencia de tamaños (size-frequency approach) se basa en las medidas de la totalidad de los líquenes de la población que crecen en la superficie. Mediante este método es posible identificar si la población corresponde a uno o varios eventos de colonización y si el talo más grande encontrado en superficie ha sido heredado o es preexistente al resto de la población.

Además se analizaron líquenes presentes en las inmediaciones del sitio pero que no se localizaban en rocas que conformaban las estructuras de origen antrópico. En cada roca se midió el diámetro de los especímenes de mayor tamaño de Rhizocarpon Rhizocarpon.

La edad de los líquenes se estimó a partir de la consideración de la tasa de crecimiento de 
Rhizocarpon Rhizocarpon. Según Garibotti et al.

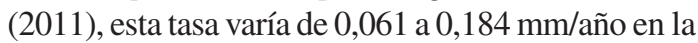
región esteparia de la Patagonia. Estos datos fueron corroborados en un reciente artículo en donde se informó los resultados obtenidos en el relevamiento del tamaño de líquenes de este subgénero en tumbas del cementerio de la ciudad de Puerto Deseado y en las superficies expuestas por el retroceso del Glaciar Túnel (Borrazzo y Garibotti 2014). La tasa de crecimiento estimada varía entre 0,061 y 0,125 mm/año (Borrazzo y Garibotti 2014:26). Se destaca que la tasa de crecimiento fue calculada a partir de la medición de especímenes de Rhizocarpon del área de estudio, ya que la ciudad de Puerto Deseado dista $40 \mathrm{~km}$ al norte del sitio Campo de Chenques. Este hecho permite considerar a la tasa de crecimiento informada por Borrazzo y Garibotti (2014) como local.

\section{Resultados}

\section{Levantamiento planimétrico en el sitio Campo de Chenques}

El relevamiento permitió contabilizar 80 estructuras de piedra y generar un mapa de sitio con coordenadas precisas, curvas de nivel y demás rasgos del entorno (Figura 4). Como se detalla en la Figura 4, las estructuras presentan una morfología en planta de forma circular o elíptica en un rango de tamaño de entre 2 y $5 \mathrm{~m}$ de largo. El promedio de tamaño de las estructuras es de $3 \pm 0,6 \mathrm{~m}$ de largo por $2,8 \pm 0,6 \mathrm{~m}$ de ancho y $0,4 \pm 0,1 \mathrm{~m}$ de alto.

El sitio se emplaza en un sector donde afloran las mismas rocas que se emplearon para la construcción de las estructuras. En todos los casos, los chenques fueron realizados con fragmentos de rocas volcánico-piroclásticas de texturas porfíricas y porfiroclásticas (ignimbritas) procedentes de las unidades litoestratigráficas del Grupo Bahía Laura (Guido 2004), sin que se registre el uso de materias primas alóctonas.

Todas las estructuras se encuentran emplazadas cercanas a la línea de costa actual, en una franja aproximada de $350 \mathrm{~m}$ de largo por $100 \mathrm{~m}$ de ancho, y entre los 5 y $10 \mathrm{msm}$ (Figura 4). A partir del levantamiento planimétrico de los chenques se observa que los mismos se encuentran formando dos grupos principales. Uno mayoritario ubicado hacia el este y otro conformado por tres chenques en el oeste (Figura 5).

\section{Liquenometría en Campo de Chenques}

Se llevó a cabo la medición de líquenes de Rhizocarpon Rhizocarpon identificados en diferentes chenques, así como en rocas del Grupo Bahía Laura que no formaban parte de estructuras antrópicas y que se hallaban fijas en las inmediaciones del sitio.

Se midieron 1.351 líquenes distribuidos en 315 rocas presentes en 51 chenques y 78 líquenes ubicados en 26 rocas dispuestas en cercanías del sitio. Los 51 chenques en donde se midió la población de líquenes (estructuras Ch 6, 7, 10, 11, $13,15-26,28-49,56,57,62,63,66,67,70,71$, 74-77; Figura 4), corresponden al $64 \%$ del total de estructuras relevadas en el sitio $(n=80)$.

A diferencia de otros sitios de referencia para Patagonia en donde se registraron especímenes de Rhizocarpon Rhizocarpon (Borrazzo y Garibotti 2014; Borrero et al. 2011), el sitio Campo de Chenques presenta un tamaño mayor y una densa colonización de líquenes.

Tanto en las rocas que conforman los chenques como en las del entorno se hallaron líquenes con talos coalescentes, lo que impidió su medición. Sin embargo, esta última situación se dio con mayor frecuencia en rocas que no formaban parte de las estructuras antrópicas. Este hecho podría ser consecuencia de un mayor tiempo de exposición de la superficie de las rocas del entorno, y por lo tanto mayor disponibilidad temporal para la colonización y crecimiento de los líquenes, en relación con el (menor) tiempo de exposición promediado por aquellas rocas que conformaban las estructuras antrópicas.

A excepción de un liquen registrado en un chenque, el cual mide $60 \mathrm{~mm}$ de diámetro, todos los casos restantes de ejemplares asociados a chenques forman parte de una distribución de frecuencia de tamaños simétrica o unimodal (Figura 6), sugiriendo que los mismos constituirían una única población coherente. Se infiere que el ejemplar de $60 \mathrm{~mm}$ habría sobrevivido al traslado de la roca utilizada al momento de construcción de la estructura. Los líquenes en los chenques son de menor tamaño que los identificados en las rocas del entorno (diámetro máximo $47 \mathrm{~mm}$ vs. $75 \mathrm{~mm}$ de diámetro, respectivamente; Figura 6), lo que permite plantear, de manera general, que los líquenes presentes en las rocas de los chenques son más jóvenes. A partir de los histogramas de tamaño es posible plantear que los líquenes presentes en las rocas de chenques 


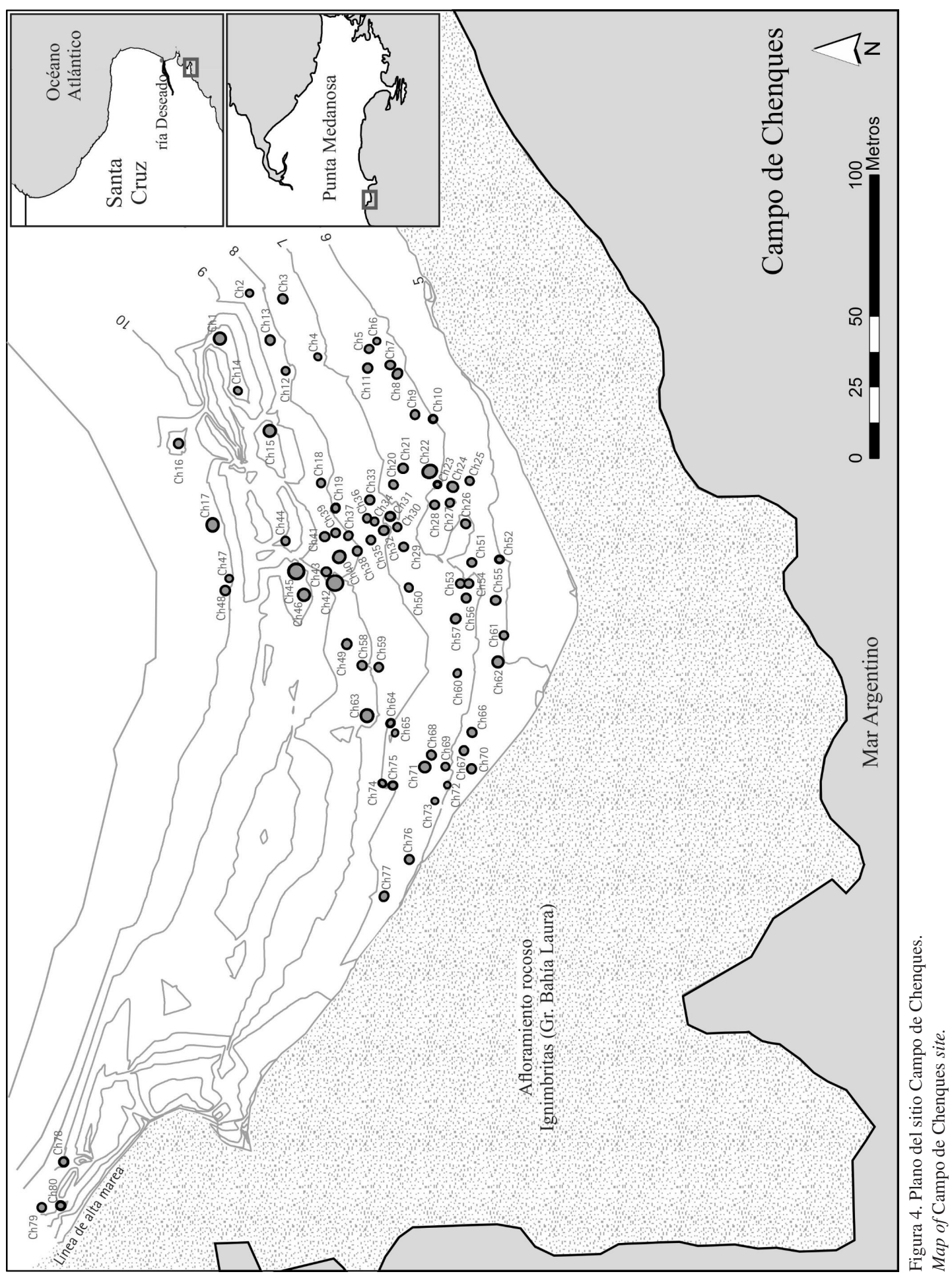



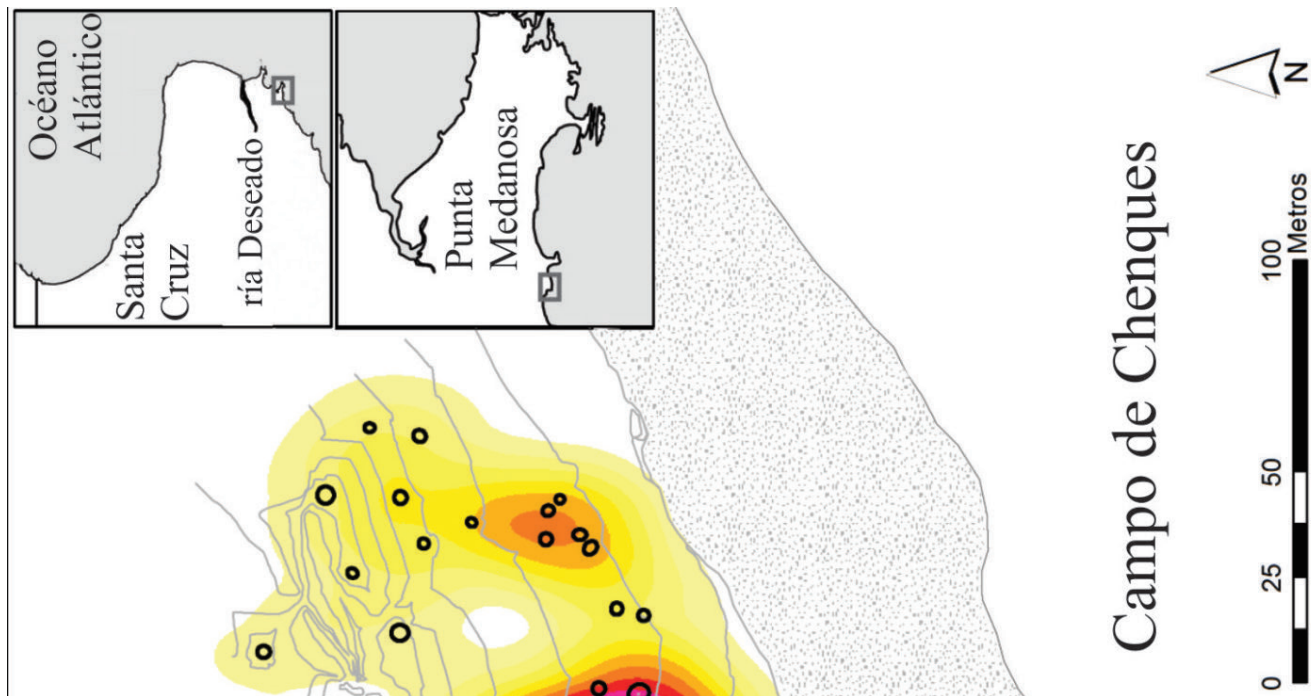


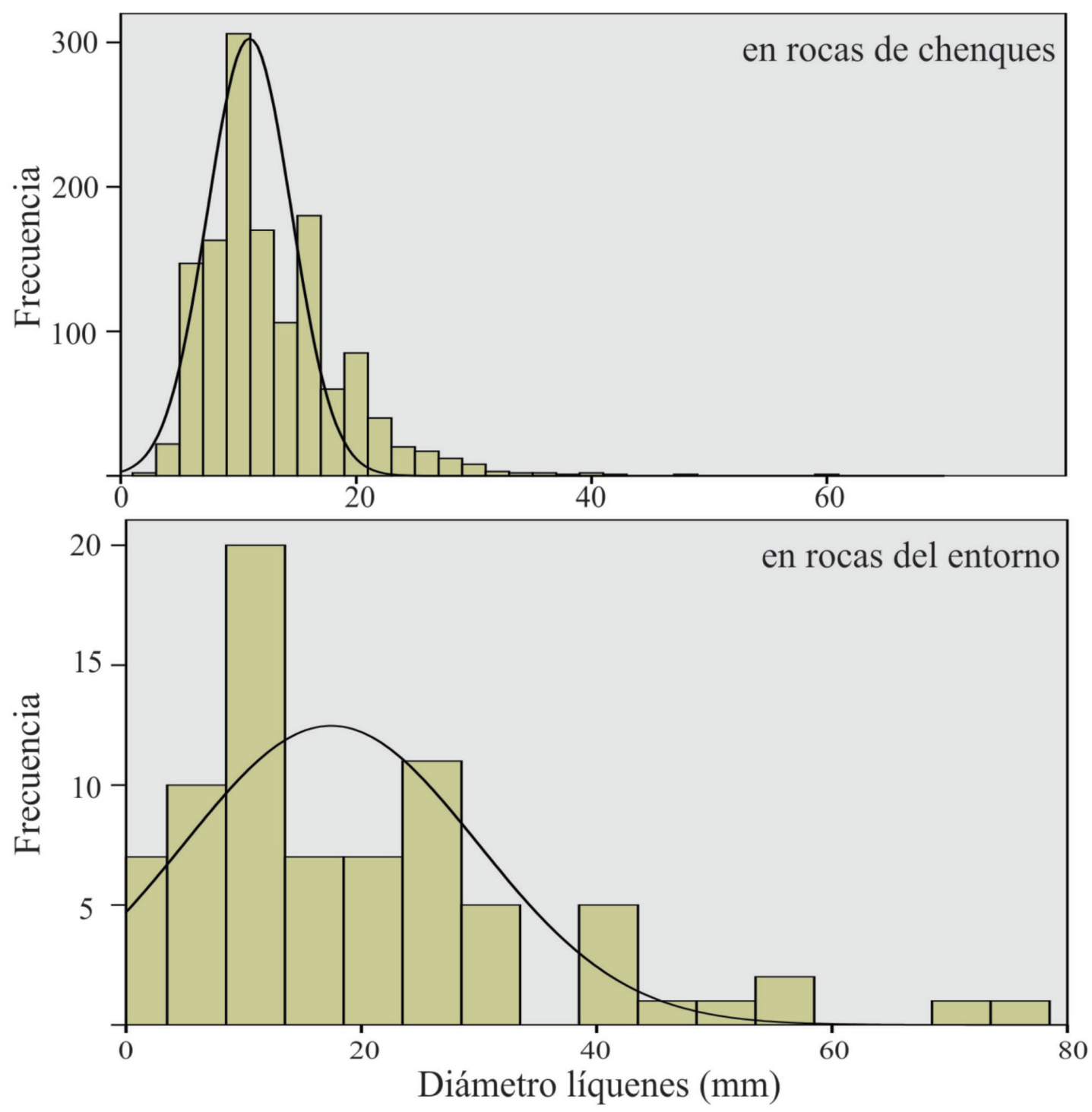

Figura 6. Histograma de los diámetros de los ejemplares de Rhizocarpon Rhizocarpon presentes en los chenques y rocas del entorno en el sitio Campo de Chenques.

Histogram of the diameters of the specimens of Rhizocarpon Rhizocarpon present on chenques and on surroundings rocks at Campo de Chenques site.

y aquellos hallados en rocas del entorno, podrían corresponder a distintas poblaciones (Figura 6).

La tasa de crecimiento de Rhizocarpon Rhizocarpon, obtenida a partir de ejemplares medidos en el cementerio de Puerto Deseado, es de 0,061 mm por año (Borrazzo y Garibotti 2014). Aplicando esta tasa al liquen más grande hallado en uno de los chenques (47 mm identificado en la estructura CCH-70; Figura 4), se estima que la edad mínima de construcción es de 770 años, aproximadamente. El liquen de $47 \mathrm{~mm}$ estaría indicando el inicio de colonización de la población de Rhizocarpon Rhizocarpon en las estructuras antrópicas.

Se realizó un análisis espacial a fin de indagar acerca de la existencia de diferencias temporales en la instalación de los líquenes en distintos sectores del sitio. Para ello se consideró la media de los diámetros de los líquenes en cada una de las 51 estructuras analizadas, así como el diámetro del liquen con mayor tamaño relevado por estructura. Se observó que los promedios del diámetro de los líquenes se presentan de forma continua o gradual 
(Figura 7). Del análisis espacial se identificó que los valores de la media, así como del diámetro del liquen de mayor tamaño por estructura se distribuyen de forma aleatoria a lo largo del sitio (Figura 7).

Se desconoce la ubicación exacta en el plano del sitio de las dos estructuras de donde se obtuvieron muestras óseas que fueron datadas por radiocarbono (CCH-1 y CCH-2; Tabla 1). A pesar de que se dispone de las ubicaciones geográficas obtenidas mediante GPS convencional, no fue posible localizarlas debido al error implícito del instrumental. De esta manera, tampoco fue posible relacionar directamente la información liquenométrica y radiocarbónica obtenida para estas dos estructuras.

\section{Discusión y Conclusiones}

A partir del relevamiento planimétrico de Campo de Chenques, mediante el empleo del GPS diferencial se logró obtener un plano preciso del sitio arqueológico, el cual no podría haber sido realizado con el uso de un GPS convencional debido al elevado margen de error de esta clase de instrumental. Gracias a la alta precisión del GPS diferencial, se logró conjugar planimétricamente la morfología de las estructuras y su distribución en el espacio con relación a las curvas de nivel.

El tamaño promedio de los chenques en el sitio es de $3 \pm 0,6 \mathrm{~m}$ de largo por $2,8 \pm 0,6 \mathrm{~m}$ de ancho. Este dato es similar al promedio general de $3,8 \mathrm{~m}$ de largo por $3 \mathrm{~m}$ de ancho que se conoce para los chenques ubicados en el área de la cuenca del lago Salitroso en la provincia de Santa Cruz (Cassiodoro 2008:97), así como para chenques registrados en otras localidades arqueológicas de la costa norte de Santa Cruz (Zilio 2015).

Es probable que un gran número de las estructuras hayan sido alteradas por saqueo, sin embargo este es un aspecto complejo de interpretar a partir del relevamiento superficial. Por lo tanto, las dimensiones actuales podrían no reflejar el tamaño original de los chenques debido a causas como el saqueo, el pisoteo de ganado, entre otras (Zilio 2013). Por otro lado, las variaciones en los tamaños podrían relacionarse con el grupo etario de las personas allí inhumadas, como fuera mencionado por Menghin y Bórmida (s/f) para el sitio.

La medición de líquenes del subgénero Rhizocarpon permitió estimar una edad mínima de construcción de las estructuras que conforman el sitio de aproximadamente 770 años. La edad mínima obtenida a partir de la técnica liquenométrica es coherente con los fechados radiocarbónicos obtenidos en muestras óseas humanas (Tabla 1). En síntesis, se dispone de dos dataciones radiocarbónicas y de una edad mínima estimada por liquenometría, las cuales ubican temporalmente a Campo de Chenques en un momento acotado del Holoceno tardío final.

A partir del análisis de la media de los diámetros de los líquenes, así como del diámetro del liquen con mayor tamaño relevado por estructura, se interpreta que posiblemente la construcción de estas en Campo de Chenques se dio, a nivel espacial, de manera aleatoria. Por otro lado, la construcción de las diferentes estructuras se habría dado de forma continua o gradual en el tiempo. Los resultados liquenométricos obtenidos abalarían la hipótesis de que los chenques en el sitio no habrían sido construidos en un único evento temporal.

Campo de Chenques es el contexto arqueológico más antiguo de Patagonia en el que se han aplicado análisis liquenométricos hasta el momento, a partir de un liquen con un talo de $47 \mathrm{~mm}$.

Si bien se ha discutido recientemente la aplicación de la liquenometría (Osborn et al. 2015), la coherencia de la fecha estimada con las edades radiocarbónicas disponibles, la procedencia local de los datos de referencia (Borrazzo y Garibotti 2014) y su coherencia con otras tasas de crecimiento calculadas para Patagonia (Garibotti et al. 2011), nos permiten considerar a esta técnica adecuada para estimar una edad mínima para la construcción de las estructuras estudiadas. Sin embargo, se prevé la realización de fechados radiocarbónicos y estudios liquenométricos por estructura que permitan evaluar la coherencia entre ambas líneas de datos en el sitio.

A partir de los análisis espaciales y cronológicos (radiocarbónicos y liquenométricos) fue posible reconocer la presencia de un importante número de estructuras de tipo chenque $(\mathrm{n}=80)$ concentradas en un sector acotado del espacio costero, así como una estimación de la antigüedad del sitio arqueológico.

Estos resultados nos permiten incorporar elementos a la discusión de las características de las formas de vida de grupos cazadores recolectores para el Holoceno tardío final. Generalmente se ha planteado que no es esperable en grupos con alta movilidad residencial la asociación a sitios con gran concentración de entierros humanos o cementerios 


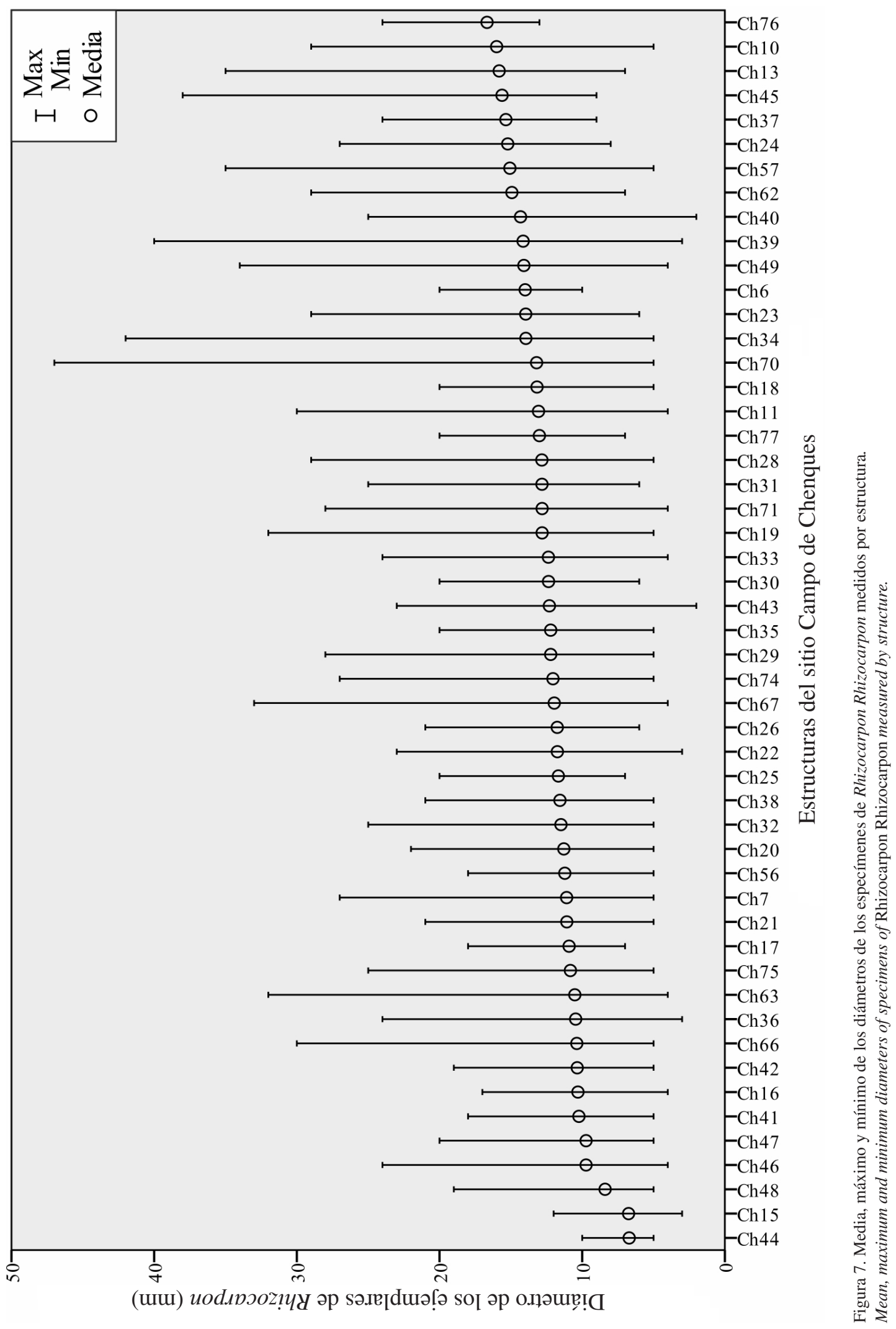


(Littleton y Allen 2007; Pardoe 1988; Walthall 1999; entre otros). En este sentido, recientemente Zilio (2015) planteó un modelo acerca de la dinámica humana durante el Holoceno Tardío en el área de la costa norte de la provincia de Santa Cruz, a partir de múltiples líneas de evidencia. Desde ca. 1.500 a.p. se habría desarrollado un período de sequía en la región el cual habría generado que los recursos ubicados en la franja costera se restringieran a determinados sectores del litoral atlántico, situados principalmente al sur de la ría Deseado, como es el caso de la localidad arqueológica Punta Medanosa (Figura 1). Debido a las circunstancias ambientales que fueron detectadas a nivel regional, se consideró que el aumento de las condiciones secas a partir de ca. 1.500 a.p., con un pico máximo de sequía alrededor de ca. 900 a.p. (Morales et al. 2009 y bibliografía allí citada), habría provocado que las poblaciones cazadoras recolectoras limitaran sus rangos de uso del espacio a sectores del interior y a la franja costera, principalmente aquella ubicada al sur de la ría Deseado. Este último sector comprendería los espacios litorales más atractivos para los asentamientos humanos debido a la disponibilidad de recursos marinos (pinnípedos, aves marinas y moluscos), así como de cuerpos de agua dulce.

En referencia a las estructuras de entierro durante el Holoceno Tardío final en la costa norte de Santa Cruz se identificó principalmente la modalidad de entierro en chenque. Los patrones distribucionales de estos entierros se presentan en general con baja frecuencia y distribuidos de forma aislada, mientras que en las localidades al sur de la ría Deseado la distribución contrasta notablemente, ya que se observan concentraciones de hasta decenas de enterratorios en espacios muy acotados, tal como ocurre en el sitio Campo de Chenques, el cual constituye el caso más extremo (Zilio 2015).

Respecto de la información paleodietaria de las cinco muestras humanas de Campo de Chenques (Tabla 1), cuatro de ellas presentan valores de $\delta^{13} \mathrm{C}$ y $\delta^{15} \mathrm{~N}$ que señalan dietas mixtas, lo que sugiere un consumo y explotación de recursos marinos y terrestres. Sin embargo, la muestra CCH-40P indica una dieta predominantemente marina. Esta última permite inferir que este individuo habría basado su dieta en recursos marinos y que, por lo tanto, debió restringir su movilidad, al menos en sus últimos años de vida, a la franja de litoral marítimo. De manera similar a los resultados isotópicos obtenidos en el sitio Campo de Chenques, otras muestras óseas humanas de la costa norte de Santa Cruz recuperadas en localidades al sur de la ría Deseado indican dietas principalmente mixtas, como así también dietas marinas, aunque en menor porcentaje (Moreno et al. 2011; Zilio 2015; Zilio et al. 2014).

Los espacios litorales ubicados al sur de la ría Deseado fueron ocupados recurrentemente en el tiempo (Zubimendi et al. 2015), y los recursos marinos se habrían explotado reiteradamente debido a que se presentaban de forma previsible, abundante y segura (principalmente pinnípedos, aves marinas y moluscos). Sin embargo, el consumo de los recursos terrestres no habría sido abandonado, como lo indican los datos isotópicos, los que sugieren la explotación complementaria de recursos terrestres y marinos. Las señales isotópicas estarían respaldando la hipótesis de la existencia de movimientos individuales, con distinto grado de duración y permanencia entre la costa y el interior. La movilidad costa-interior en el sector sur de la ría Deseado y en algunas ocasiones limitada a la franja de costa por estancias prolongadas (evidenciada por las señales isotópicas que indican dietas predominantemente marinas), habría generado la concentración de los entierros en chenque en determinados sectores de la costa, consecuencia del mayor tiempo de permanencia en estos espacios. De esta manera, la mayor frecuencia de estructuras mortuorias en el sector sur de la ría Deseado se relacionaría con una mayor productividad de estas costas, una redundancia ocupacional y una reducción de la movilidad residencial durante el Holoceno tardío final (Zilio 2015).

No excluimos otras causas o razones para explicar el origen de este tipo de registro representado por una alta contigüidad espacial de entierros, el cual de seguro posee relaciones con cuestiones sociales cuyos elementos de contrastación aún no han podido ser reconocidos.

Agradecimientos: Agradecemos a Grato $\mathrm{Coccoz}$ por facilitar el GPS diferencial y colaborar en la realización del plano del sitio. A Luli y Sergio Vidal de la Ea. El Amanecer. A la Dra. Karen Borrazzo por sus comentarios y sugerencias, los que permitieron mejorar este artículo. A la Dra. Vilma Rosato (LEMIT) por la determinación taxonómica de los líquenes. A la Universidad Nacional de la Plata (UNLP) y al Consejo Nacional de Investigaciones Científicas y Técnicas (CONICET). Además, agradecemos a los dos evaluadores anónimos y a los editores cuyos comentarios posibilitaron su publicación. 


\section{Referencias Citadas}

Amado Reino, X. 1999. El GPS en Arqueología: Introducción y ejemplos de uso. TAPA Traballos en Arqueoloxía da Paisaxe 15:5-13.

Barberena, R. 2002. Los Límites del Mar. Isótopos Estables en Patagonia Meridional. Sociedad Argentina de Antropología, Buenos Aires.

Bórmida, M. 1953-1954. Los antiguos patagones. Estudio de craneología. Runa 6:5-96.

Borrazzo, K. e I.A. Garibotti 2014. Nuevos datos de referencia para la tasa de crecimiento de Rhizocarpon subg. Rhizocarpon en Patagonia. Magallania 42:199-203.

Borrero, L.A., K. Borrazzo, I.A. Garibotti y M.C. Pallo 2011. Concentraciones de pilas de roca en la cuenca superior del río Santa Cruz (Argentina). Magallania 39:193-206.

Borrero, L.A., R. Guichón, R. Tykot, J. Kelly, A. Prieto y P. Cárdenas 2001. Dieta a partir de isótopos estables en restos óseos humanos de Patagonia austral. Estado actual y perspectivas. Anales Instituto de la Patagonia 29:119-127.

Bronk Ramsey, C. y S. Lee 2013. Recent and planned developments of the program OxCal. Radiocarbon 55:3-4.

Cassiodoro, G. 2008. Movilidad y Uso del Espacio de CazadoresRecolectores del Holoceno Tardío: Estudio de la Variabilidad del Registro Tecnológico en Distintos Ambientes del Noroeste de la Provincia de Santa Cruz (Argentina). Tesis para optar al grado de Doctor en Filosofía y Letras, Facultad de Filosofía y Letras, Universidad de Buenos Aires, Buenos Aires.

Castro, A.S. 2001. Informe de actividades de campo 2001. Universidad Nacional de La Plata. Informe remitido a la Secretaría de Cultura de la Provincia de Santa Cruz. Manuscrito en posesión del autor.

Castro, A.S. y J.E. Moreno 2000. Noticia sobre enterratorios humanos en la costa Norte de Santa Cruz (Patagonia Argentina). Anales del Instituto de la Patagonia 28:225-232.

Castro, A.S., J.E. Moreno y C. Peña 2001. Los entierros de la localidad Punta Medanosa y Campo de Chenques: distribución y variabilidad. Ponencia presentada en el Taller Internacional de Arqueología de Entierros Humanos en Fuego Patagonia, Puerto Natales.

Colonese, A.C., M. Collins, A. Lucquin, M. Eustace, Y. Hancock, R. Ponzoni, A. Mora, C. Smith, P. De Blasis, L. Figuti, V. Wesolowski, C. Plens, S. Eggers, D. Scunderlick Eloy, A. Gledhill y O. Craig 2014. Long-term resilience of late Holocene coastal subsistence system in southeastern South America. PloS ONE 9:1-13.

De Niro, M.J. 1985. Postmortem preservation and alteration of in vivo bone collagen isotope ratios in relation to paleodietary reconstruction. Nature 317:806-809.

Favier Dubois, C., F. Borella y R.H. Tykot 2009. Explorando tendencias en el uso humano del espacio y los recursos en el litoral rionegrino (Argentina) durante el Holoceno medio y tardío. En Arqueología de la Patagonia. Una Mirada desde el Último Confin, editado por M. Salemme, F. Santiago, M. Álvarez, E. Piana y M. Vázquez y M.E. Mansur, pp. 985-998. Utopías, Ushuaia.
Garibotti, I.A., K. Borrazzo y L.A. Borrero 2011. Aplicación de técnicas liquenométricas en la arqueología de Patagonia utilizando Rhizocarpon subgénero Rhizocarpon. En Bosques, Montañas y Cazadores. Investigaciones Arqueológicas en Patagonia Meridional, editado por L.A. Borrero y K. Borrazzo, pp. 85-103. Dunken, Buenos Aires.

Gómez Otero, J. 2006. Dieta, Uso del Espacio y Evolución en Poblaciones Cazadoras-Recolectoras de la Costa CentroSeptentrional de Patagonia durante el Holoceno Medio y Tardío. Tesis para optar al grado de Doctor en Filosofía y Letras, Facultad de Filosofía y Letras, Universidad de Buenos Aires, Buenos Aires.

Goñi, R., G. Barrientos y S. García Guraieb 2005. Análisis preliminar del material óseo humano recuperado en el Chenque $\mathrm{n}^{\circ}$ 1, sitio Campo de Chenques, Punta Medanosa (provincia de Santa Cruz). Informe técnico, Instituto Nacional de Antropología y Pensamiento Latinoamericano. Manuscrito en posesión de los autores.

Guichón, R., L.A. Borrero, A. Prieto, P. Cárdenas y R. Tykor 2001. Nuevas determinaciones de isótopos estables para Tierra del Fuego. Revista Argentina de Antropología Biológica 3:113-126.

Guido, D.M. 2004. Subdivisión litofacial e interpretación del volcanismo jurásico (Grupo Bahía Laura) en el este del Macizo del Deseado, provincia de Santa Cruz. Revista de la Asociación Geológica Argentina 59:727-742.

Hogg, A.G., Q. Hua, P.G. Blackwell, M. Niu, C.E. Buck, T.P. Guilderson, T.J. Heaton, J.G. Palmer, P.J. Reimer, R.W. Reimer, C.S.M. Turney y S.R.H. Zimmerman 2013. SHCal13 Southern Hemisphere calibration, 0-50,000 cal yr BP. Radiocarbon 55:1889-1903.

Littleton, J. y H. Allen 2007. Hunter-gatherer burials and the creation of persistent places in southeastern Australia. Journal of Anthropological Archaeology 2:283-298.

Menghin, O.F.A. y M. Bórmida s/f. Arqueología de la costa patagónica. Manuscrito en posesión de los autores.

Morales, M., R. Barberena, J.B. Belardi, L. Borrero, V. Cortegoso, V. Durán, A. Guerci, R. Goñi, A. Gil, G. Neme, H. Yacobaccio y M. Zárate 2009. Reviewing human-environment interactions in arid regions of southern South America during the past 3000 years. Palaeogeography, Palaeoclimatology, Palaeoecology 281:283-295.

Morano Büchner, C. y J.C. Aravena 2013. Lichenometric analysis using genus Rhizocarpon, section Rhizocarpon (Lecanorales: Rhizocarpaceae) at Mount San Lorenzo, southern Chile. Revista Chilena de Historia Natural 86:465-473.

Moreno Jiménez, A. 2005. Modelización cartográfica de densidades mediante estimadores Kernel. Treballs de la Societat Catalana de Geografía 30:155-170.

Moreno, E., A.F. Zangrando, A. Tessone y A. Castro y H.O. Panarello 2011. Isótopos estables, fauna y artefactos en el estudio de los cazadores recolectores de la Costa Norte de Santa Cruz. Magallania 39:265-276.

Osborn, G., D. McCarthy, A. LaBrie y R. Burke 2015. Lichenometric dating: Science or pseudo-science? Quaternary Research 83:1-12. 
Pardoe, C. 1988. The cemetery as a symbol: The distribution of prehistoric aboriginal grounds in Southeastern Australia. Archaeology in Oceania 23:1-16.

Santana Sagredo, F., M. Hubbe y M. Uribe 2015. Isotopic evidence for marine consumption and mobility in the Atacama Desert (Quillagua, Northern Chile). International Journal of Osteoarchaeology 26:476-489.

Schoeninger, M.J., M.J. De Niro y H. Tauber 1983. Stable nitrogen isotope ratios of bone collagen reflect marine and terrestrial components of prehistoric human diet. Science 220:1381-1383.

Suby, J.A., R. Guichón y F.A. Zangrando 2009. El registro biológico humano de la costa meridional de Santa Cruz. Revista Argentina de Antropología Biológica 11:109-124.

Tessone, A. 2010. Arqueología y Ecología Isotópica. Estudio de Isótopos Estables de Restos Humanos del Holoceno Tardío en Patagonia Meridional. Tesis para optar al grado de Doctor en Filosofía y Letras, Facultad de Filosofía y Letras, Universidad de Buenos Aires, Buenos Aires.

Tomczak, P. 2003. Prehistoric diet and socioeconomic relationships within the Osmore valley of southern Peru. Journal of Anthropological Archaeology 22:262-278.

Walthall, J. 1999. Mortuary behavior and early Holocene land use in the North American midcontinent. North American Archaeologist 20:1-30.
Yesner, O., M. Figuerero Torres, R. Guichón y L.A. Borrero 1991. Análisis de isótopos estables en esqueletos humanos: confirmación de patrones de subsistencia etnográficos para Tierra del Fuego. Shincal 3(2):182-191.

Zilio, L. 2013. Chenques en Patagonia Centro-meridional: análisis de los patrones de distribución espacio-temporales. Comechingonia 17:237-254.

Zilio, L. 2015. Prácticas Mortuorias en La Costa Norte de Santa Cruz: Arqueología de Sociedades Cazadoras Recolectoras en Paisajes Costeros de la Patagonia Argentina. Tesis para optar al grado de Doctor en Ciencias Naturales, Facultad de Ciencias Naturales y Museo, Universidad Nacional de La Plata, La Plata.

Zilio, L., F. Górdon, M. Béguelin y A.S. Castro 2014. Paleodietas humanas en el sur del Golfo San Jorge (provincia de Santa Cruz) a partir del análisis de isótopos estables. Revista Argentina de Antropología Biológica 16:51-64.

Zilio, L., M.A. Zubimendi y H. Hammond 2013. Chenques en un paisaje costero: análisis espacial de estructuras de entierro en Punta Medanosa. Anuario de Arqueología 5:253-267.

Zubimendi, M.A., P. Ambrústolo, L. Zilio y A. Castro 2015. Continuity and discontinuity in the human use of the north coast of Santa Cruz (Patagonia Argentina) through its radiocarbon record. Quaternary International 356:127-146.

\section{Nota}

1 Los fechados radiocarbónicos fueron calibrados utilizando el programa OxCal 4.2 (Bronk Ramsey y Lee 2013). La calibración se llevó a cabo en años a.p., con dos desvíos estándar. Se empleó la curva de calibración para el Hemisferio Sur (SHCal13) presentada por Hogg et al. (2013). 